\title{
Recurrent Vomiting and Epigastric Pain Attacks in an Adult Patient With Colonic Malrotation
}

\author{
Yucel Ustundaga,, Ismail Serifoglu ${ }^{\mathrm{b}}$, Oge Tascilar $^{\mathrm{c}}$
}

\begin{abstract}
Intestinal malrotation occurs when there is a disruption of normal embryological development of bowel. Recurrent abdominal pain due to bowel obstruction associated with adhesions is the most common clinical presentation. The majority of patients present with clinical findings in childhood. Its diagnosis is mainly based on radiologic imaging methods. Surgery is the treatment of choice for these patients. The success of treatment and prevention of complications depends on an accurate diagnosis and exact localization of colonic and intestinal anatomy. Herein, we report a 39-year-old man having intermittent attacks of nausea, vomiting and epigastric pain starting from the onset of childhood. His last attack was associated with acute pancreatitis as well. He was diagnosed with colonic malrotation on radiological examinations. Surgical resection of Ladd's bands was successful in relieving further attacks of bowel obstruction and acute pancreatitis. We discussed this case with a short review of relevant literature.
\end{abstract}

Keywords: Colonic malrotation; Proximal bowel obstruction; Acute panreatitis; Radiologic diagnosis; Surgical treatment

\section{Introduction}

Intestinal obstruction due to colonic malrotation is a challenging diagnosis and a very rare presentation. Its diagnosis is mainly based on radiologic imaging methods [1]. The presence of radiological signs such as the whirl sign of mes-

\footnotetext{
Manuscript accepted for publication March 4, 2014

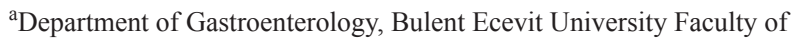
Medicine, Zonguldak, Turkey

${ }^{\mathrm{b}}$ Department of Radiology, Bulent Ecevit University Faculty of

Medicine, Zonguldak, Turkey

${ }^{\mathrm{c}}$ Department of General Surgery, Bulent Ecevit University Faculty of Medicine, Zonguldak, Turkey

${ }^{\mathrm{d}}$ Corresponding author: Yucel Ustundag, Department of

Gastroenterology, Faculty of Medicine, Bulent Ecevit University,

Zonguldak, Turkey. Email: yucelustundag@yahoo.com.tr
}

doi: http://dx.doi.org/10.14740/jmc1742w enteric vasculature and left sided cecum on abdominal computerized tomography examination and the clinical signs of intestinal obstruction are of diagnostic importance for this disease entity $[1,2]$. Surgery is the treatment of choice for these patients [3]. The success of treatment and prevention of complications depends on an accurate diagnosis and exact localization of colonic and intestinal anatomy [4]. Herein, we report a 39-year-old man having intermittent attacks of nausea, vomiting and epigastric pain leading to a diagnosis of intestinal and colonic malrotation. We presented our case with detailed clinical, radiological and surgical findings and summarized the relevant literature.

\section{Case Report}

A 39-year-old man was admitted to our hospital with the complaints of vomiting and acute epigastric pain. He noticed that he vomited digested food and bilious contents postprandially for the last few weeks. He also said he had been suffering from intermittent abdominal pain since his childhood. He had no medication use for any known illness. He also never consumed ethanol. Physical examination revealed

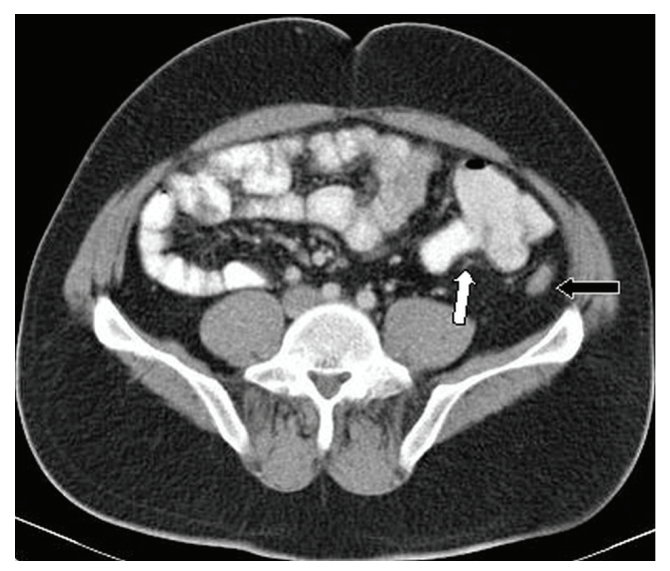

Figure 1. In axial CT imaging at infraumbilical level cecum and appendix was seen in the left lower abdominal quadrant (white arrow). Collabed dessendan colon was seen in lateral of cecum (black arrow). 


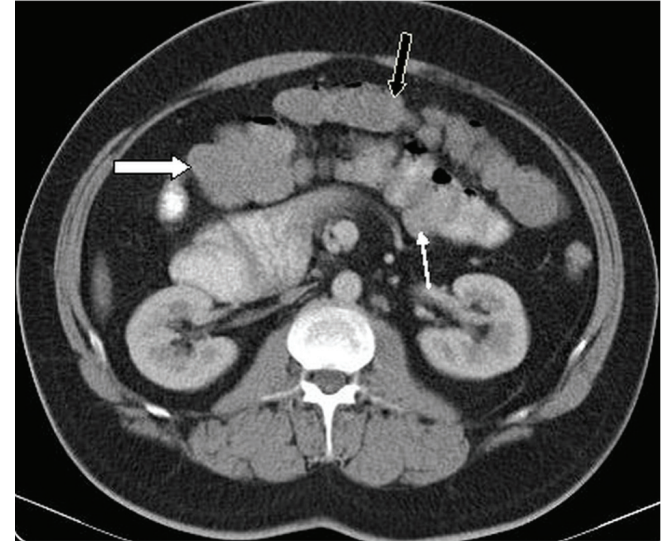

Figure 2. In axial CT image the ascending colon (thin white arrow) had a turn to the right side of the abdomen and thereafter it joined to transverse colon (black arrow) after making hepatic flexura (white arrow) nearly at the second part of the duodenum.

epigastric tenderness and abdominal distension. Laboratory studies showed a normal hemogram with a slightly elevated C-reactive protein. His blood biochemistry revealed elevated levels of serum transaminases (alanine aminotransferase, 97 $\mathrm{U} / \mathrm{L}$; aspartate aminotransferase, $42 \mathrm{U} / \mathrm{L}$ ), amylase $465 \mathrm{U} / \mathrm{L}$ and lipase $734 \mathrm{U} / \mathrm{L}$. The other tests of blood biochemistry including glucose, bilirubin, alkaline phosphatase, g-glutamyltransferase, triglycerides and electrolytes were completely normal. An abdominal computed tomography (CT) examination revealed that cecum and appendix were seen in the left lower abdominal quadrant and the ascending colon was lying parallel to descending colon till to the level of left kidney (Fig. 1). At that level, the ascending colon had a turn

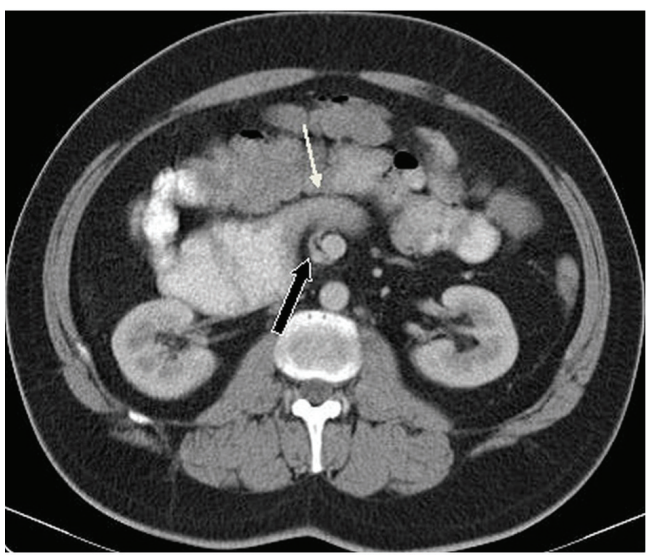

Figure 3. Axial CT image demonstrates transition point (thin white arrow) between dilated second part and collabed third part of duodenum where the ascending colon passes from the right to the left of mid-abdomen. Additionally in this level also seen whirl sign (black arrow) due to rotation of superior mesenteric vein (SMV) leftward on superior mesenteric artery (SMA).

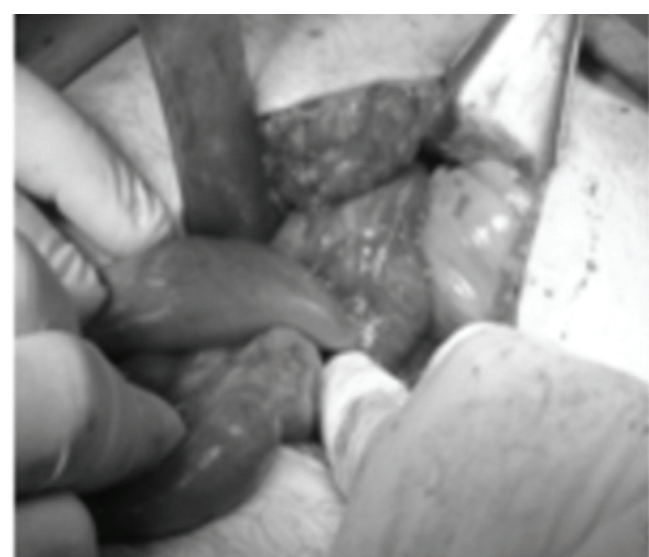

Figure 4. On surgical exploration, there was distension in the stomach and throughout the duodenum and, there were Ladd's bands crossing and squeezing especially the distal part of the duodenum.

to the right side of the abdomen and thereafter it joined to transverse colon after making hepatic flexura nearly at the second part of the duodenum. There was a sudden and obvious decrease in the luminal diameter of second and third part of the duodenum where the ascending colon passes from the right to the left of mid-abdomen. Proximally duodenum is noted to be dilated. Additionally, we noticed the existence of whirl sign due to rotation of superior mesenteric vein (SMV) leftward on superior mesenteric artery (SMA) (Fig. 2, 3). The gallbladder, intrahepatic and extrahepatic bile ducts were seen normally on magnetic resonance cholangiography.

The patient was referred to operating room. On exploration, there was distension in the stomach and throughout the duodenum and, there were Ladd's bands crossing and squeezing especially the distal part of the duodenum. The third and fourth parts of the duodenum were found to reside intraperitoneally, rather than their expected natural place in the retroperitoneum. Also, the cecum, appendix and ascending colon were found to reside completely in the left abdominal space (Fig. 4). The bands around duodenum were excised surgically. The other abdominal organs were in their normal anatomical position. A diagnosis of incomplete midgut malrotation was made in this patient.

\section{Discussion}

Intestinal malrotation has been considered primarily a disease of infancy or childhood. However, it can be diagnosed in adults with variable clinical signs and symptoms. Seven different types and degrees of intestinal malrotation were known according to complete or partial failures of rotation and abnormalities affecting the duodenojejunal loop, the cecocolic loop, or both. As in our patient, the most common type is type Ia and the duodenum and large bowel stop rota- 
tion after $90^{\circ}$ in that type, so that the proximal small bowel, including the duodenojejunal junction, lies on the right and cecum lies on the left [5]. After 12th gestational week, fixation of intestine throughout peritoneal bands (Ladd's bands) to the posterior body wall occurs. In the setting of colonic malrotation, the tightening of these bands may lead to duodenal obstruction as occurred in our case [6]. Due to obstruction, high intra duodenal pressure and reflux of duodeno-biliary contents can complicate as recurrent pancreatitis attacks which is a very rare reason of pancreatitis [7].

Intestinal obstruction due to cecal malrotation can be difficult to diagnose clinically. In clinics, the most frequent symptom is abdominal pain. Other symptoms include nausea, vomiting, constipation, abdominal distension diarrhea, malabsorption, peritonitis and septic shock $[4,8]$. Our patient presented with chronic recurrent abdominal pain and vomiting. In adult patients, these clinical symptoms can be mistaken for peptic ulcer, irritable bowel syndrome, pancreatic and biliary disorders. Because of these nonspecific clinic symptoms, the diagnosis of malrotation primarily lies on radiologic examinations. In infancy, the main diagnostic approach is plain abdominal film and ultrasonography (USG). Although the plain abdominal radiographs do not have specific findings indicating malrotation, the dilatation of bowel segments can be easily seen on these graphs. If this finding exists, there needs to be prompt further investigation. Ultrasonography is a primarily diagnostic modality in infants. On USG examination, the dilated duodenal segments, whirl sign between rotated duodenum, SMV and SMA, and fixed midline duodenum can be noticeable findings [9]. The CT is the primary diagnostic method of choice in adult patients. On CT examination, whirlpool sign is a good indicator of intestinal malrotation even in the absence of volvulus [9]. Furthermore, abnormally positioned cecum and ascending colon in the left lower quadrant also will be clearly seen on CT examination. Due to Ladd's bands, duodenal obstruction can occur and transition point between dilated and non-dilated bowel segments will be apparently seen on CT imaging as well. Surgical intervention is definitely treatment option for colonic malrotation. Ladd's procedure is a choice of reasonable treatment and during this operation, an appendectomy may be performed or not.

As a conclusion, this case reminds us the importance of a tedious radiological evaluation in the setting of recurrent abdominal pain and vomiting in daily clinical practice.
Clinicians should be aware of colonic malrotation presenting with symptoms of intestinal obstruction and pancreatitis even in adulthood. This is very much important for proper treatment decision and to avoid surgical complications in these patients.

\section{Conflict of Interest}

This manuscript has not been previously submitted for publication in another journal at this time and has not been previously published. All the authorship are aware of and approve the manuscript being submitted to this journal.

\section{References}

1. Lampl B, Levin TL, Berdon WE, Cowles RA. Malrotation and midgut volvulus: a historical review and current controversies in diagnosis and management. Pediatr Radiol. 2009;39(4):359-366.

2. van den Hoven I, Roumen RM. Chronic recurrent crampy abdominal pain owing to partial intestinal malrotation. Surgery. 2010;147(6):893-894.

3. Hinkle JK, Smith TR. Malrotation with volvulus of the transverse colon and duodenal obstruction secondary to Ladd's bands. Clin Imaging. 2008;32(1):65-68.

4. Gamblin TC, Stephens RE, Jr., Johnson RK, Rothwell M. Adult malrotation: a case report and review of the literature. Curr Surg. 2003;60(5):517-520.

5. Stringer DA, Jamieson D. Small bowel. In: Stringer DA, Babyn PS, editors. Pediatric gastrointestinal imaging and intervention. 2nd ed. London: B.C. Decker Inc; 2000. pp. 311-475.

6. Nehra D, Goldstein AM. Intestinal malrotation: varied clinical presentation from infancy through adulthood. Surgery. 2011;149(3):386-393.

7. Just JD, Bailey RJ. Duodenal obstruction from congenital bands: an unusual cause of pancreatitis. Can J Gastroenterol. 1996;10(7):449-450.

8. Burke MS, Glick PL. Gastrointestinal malrotation with volvulus in an adult. Am J Surg. 2008;195(4):501-503.

9. Kapfer SA, Rappold JF. Intestinal malrotation-not just the pediatric surgeon's problem. J Am Coll Surg. 2004;199(4):628-635. 